\title{
Hybrid of convolutional neural network algorithm and autoregressive integrated moving average model for skin cancer classification among Malaysian
}

\author{
Chee Ka Chin', Dayang Azra binti Awang Mat ${ }^{2}$, Abdulrazak Yahya Saleh ${ }^{3}$ \\ ${ }^{1,2}$ Department of Electrical and Electronic, Faculty of Engineering, Universiti Malaysia Sarawak, 94300 Kota Samarahan, \\ Sarawak, Malaysia \\ ${ }^{3}$ FSKPM Faculty, Universiti Malaysia Sarawak (UNIMAS), Kota Samarahan 94300, Sarawak, Malaysia
}

\begin{tabular}{|c|c|}
\hline Article Info & ABSTRACT \\
\hline Article history: & Skin cancer is a widely spreading cause of mortality among the people \\
\hline Received Dec 28, 2020 & $\begin{array}{l}\text { specifically living on or near the equatorial belt. Early detection of skin cancer } \\
\text { significantly improves the recovery prevalence and the chance of surviving. }\end{array}$ \\
\hline Revised May 14, 2021 & Without the assist of computer-aided decision (CAD) system, skin cancer \\
\hline Accepted May 29, 2021 & $\begin{array}{l}\text { classification is the challenging task for the dermatologist to differentiate the } \\
\text { type of skin cancer and provide the suitable treatment. Recently, the }\end{array}$ \\
\hline Keywords: & $\begin{array}{l}\text { development of machine learning and pretrained deep neural network (DNN) } \\
\text { shows the tremendous performance in image classification task which also }\end{array}$ \\
\hline ARIMA & provide the promising performance in medical field. However, these machine \\
\hline Classification & learning methods cannot get the deep features from network flow which \\
\hline Convolutional neural network & $\begin{array}{l}\text { resulting in low accuracy and the pretrained DNN has the complex network } \\
\text { with a huge number of parameters causes the limited classification accuracy. }\end{array}$ \\
\hline Deep neural network & This paper focuses on the classification of skin cancer to identify whether it is \\
\hline Skin cancer & $\begin{array}{l}\text { basal cell carcinoma, melanoma or squamous cell carcinoma by using the } \\
\text { development of hybrid convolutional neural network algorithm and } \\
\text { autoregressive integrated moving average model (CNN-ARIMA). The CNN- } \\
\text { ARIMA model was trained and found to produce the best accuracy of } \\
92.25 \% \text {. }\end{array}$ \\
\hline
\end{tabular}

This is an open access article under the CC BY-SA license.

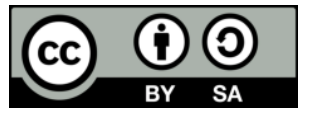

Corresponding Author:

Chee Ka Chin

Department of Electrical and Electronic, Faculty of Engineering

Universiti Malaysia Sarawak

94300 Kota Samarahan, Sarawak, Malaysia

Email: 19020098@siswa.unimas.my

\section{INTRODUCTION}

In National Cancer Registry data, Malaysia's reported that skin cancers account for $2.6 \%$ cancer cases in the country which considers as minor cases in Malaysia [1]. Based on data collected from Dermatology Clinic in Hospital Kuala Lumpur from 2006 to 2014 [2], the most common type of skin cancer is basal cell carcinoma with $34.9 \%$, followed by cutaneous lymphoma with $25.7 \%$ and squamous cell carcinoma in $20.6 \%$ cases while melanoma is the least common cases which only $5.4 \%$ of the patients diagnosed. Even though there are less skin cancer cases in Malaysia, this disease still needs to be concerned due to skin cancer consider as most predominant type of cancer in worldwide [3].

Dermatologists often adopt various diagnosis guide for skin lesion such as ABCDE rule (A-stands for asymmetrical shape, B-irregular borders of lesion, C-uneven distribution of color, D-diameter, and E-a presence of evolution) [4], [5] and 7-point checklist [6], [7] but need to be further examined via 
epiluminescence microscopy (ELM). Garnavi et al. described that there is an improvement of 5-30\% in the detection while using ELM as compared to the examination of naked eye [8], [9]. Therefore, it is possible to assist dermatologists via computer-aided diagnosis (CAD) system for analyzing the pigmented skin lesions efficiently with the development of ELM through automatic and quantitative assessment of the skin lesion in clinical practice.

Recently, convolutional neural network (CNN) allowed computers to outperform in the classification tasks of skin cancer as compare to the machine learning due to the CNN has ability to handle the large and complex datasets that produce supremacy performance in terms of accuracy [10]. This can be proved through the study about the artificial intelligence (AI) program that trained on nearly 130,000 images of moles, rashes, and lesions using a technique known as deep learning which provide "at least" 91 percent accuracy [11]. The benefit of CNN is this architecture able to classify the skin lesions according to the highlevel features instead of the conventional method that incorporate the visual information of low-level dermoscopic that needed a segmentation step before the extraction of those features [12].

In addition, autoregressive integrated moving average (ARIMA) model which known as the BoxJenkins method [13] is one of the machine learning algorithms also show the great performance in medical cases forecasting recently through the statistical analysis [14]. Therefore, the hybrid approach of CNN and ARIMA has been proposed for skin cancer classification problem in this paper. This approach believe that will be performed efficiently on a reliable skin cancer classification with the ability to differentiate between three different common types of skin cancer such as melanoma, basal cell carcinoma and squamous cell carcinoma. Actually, the aim of this paper is to evaluate the hybrid of CNN algorithm and ARIMA model performance for skin cancer classification among Malaysian.

\section{RELATED WORKS}

Recently, researchers around the world focus on solving the classification problems in medical field especially skin cancer classification by using the machine learning and deep learning approaches. Codella $e t$ al. [15] depicted the combination of deep learning and sparse coding as feature extractor while the fused support vector machine (SVM) algorithm was used for classification task. The beauty of this paper is the adoption of the deep learning in feature extraction task which able to increase time and space complexity of the model. After that, an improvement approach has been introduced by same author in terms of the automated segmentation, multi-contextual analysis of the extracted features and additional machine learning techniques for classification task [16].

In Ozkan and Koklu [17] proposed a skin lesion classification system by applying four machine learning methods to classify the lesion into melanoma, abnormal, and normal. In this work, the highest accuracy percentage of $92.50 \%$ was performed by the artificial neural networks (ANN). This obviously shows that machine learning techniques still consider as low accuracy performance when compare to ANN due to the machine learning methods unable to get the deep features from network flow. Oliveira et al. [18] used used the ABCD rule and the Box-Counting approach as attribute extraction descriptors and support vector machine (SVM) as classifier for the identification pigmented skin lesions in macroscopic images. The best performance was achieved by SVM with histogram intersection kernel give the accuracy of $79.01 \%$ at the classification between nevi and seborrheic keratosis.

For the utilization of deep learning approach in skin cancer classification, Emara et al. [19] proposed the modified Inception-v4 architecture which was also a CNN architecture to classify skin lesion through imbalance HAM 10000 skin cancer dataset [20]. The enhancement of this work was employing the feature reuse using long residual connection to improve classification performance with the achievement of high accuracy, $94.70 \%$ but this pretrained DNN has the complex network with a huge number of parameters that may causes the limited classification accuracy. Mukherjee et al. [21] using CNN methods named as CNN malignant lesion detection (CMLD) architecture to analyze the classification accuracy performance of malignant melanoma. The best performance in this work was achieved with accuracy of $90.58 \%$ when using Dermofit dataset for model training and testing. Recently, Kassem et al. [22] used modified GoogleNet by replacing last two layers with multiclass support vector machine (SVM) with transfer learning to classify the skin lesion. This hybrid approach achieved classification performance with accuracy, sensitivity, specificity, and precision are $94.92 \%, 79.80 \%, 97.00 \%$, and $80.36 \%$.

Since ARIMA model shows the outstanding performance in forecasting tasks, researchers tend to use hybridisation methods for enhancement due to its outstanding performance in solving a lot of engineering problems. Wang et al. [23] developed the hybrid model of SVM and ARIMA (SVM-ARIMA) to the garlic short-term price and outperformed the ARIMA and SVM models. Ma et al. [24] proposed hybrid of multilayer perceptron (MLP) neural network with ARIMA model which named as NN-ARIMA and hybrid of multidimensional support vector regression (MSVR) with ARIMA model which known as MSVR-ARIMA

Int J Artif Intell, Vol. 10, No. 3, September 2021: 707 - 716 
for network-wide traffic state prediction. MLP neural network and MSVR were implemented to capture the network-scale co-movement pattern of all traffic flows while ARIMA was utilized to postprocess the residual time series out of neural network in this paper. Suhermi et al. [25] employed the hybrid deep neural network (DNN) and ARIMA model for roll motion prediction. This proposed approach is based on the supremacy performance of ARIMA and artificial neural network (ANN) models in linear and non-linear modelling. In addition, the advance hybrid approach has been yielded by Ji et al. [26]. The authors introduced the hybrid of ARIMA model, CNN and long short-term memory network (LSTM) to forecast the future prices of carbon. The idea behind of this hybrid method is ARIMA used for linear features capturing. CNN utilized for hierarchical data structure capturing while LSTM utilized for long-term dependencies capturing in the data. The beauty of these papers were the empirical results of hybrid model performed better than that of nonhybrid model and proven that the hybrid method quite effective in improvement of forecast accuracy.

\section{METHODOLOGY}

In this paper, a new technique has been introduced for skin cancer classification using hybridisation technique by combining CNN algorithm and ARIMA model. Our technique has been implemented and validated. From the CNN-ARIMA model, CNN algorithm will be used to extract the features of skin cancer and ARIMA model behave as classifier to classify the skin cancer based on three types common skin cancer which consists of melanoma, basal cell carcinoma and squamous cell carcinoma [27].

\subsection{Dataset}

The dataset used in this paper was collected from The International Skin Imaging Collaboration (ISIC) via Kaggle website [28]. This dataset consists of 2357 RGB dermoscopic images in JPEG format which categorized in 9 different skin cancer diseases based on the classification taken from ISIC. These images were very clean with high resolution. Therefore, the dataset from ISIC is suitable for deep learning implementation. Besides that, there were also different image resolution from this source since the archive combines many sub datasets. In this paper, there were 995 images which comprised of 3 different classes in Figure 1 such as melanoma, basal cell carcinoma and squamous cell carcinoma were selected due to these three types of skin cancers considered as common in Malaysia. Figure 1 illustrates the selected skin cancer types that used in this work such as Melanoma in Figure 1(a), Basal cell carcinoma in Figure 1(b) and Squamous cell carcinoma in Figure 1(c).

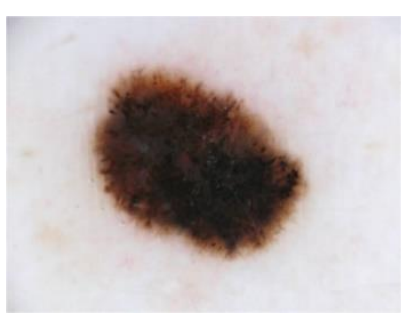

(a)

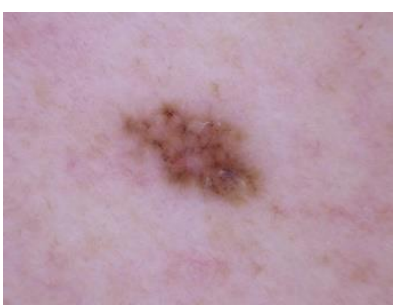

(b)

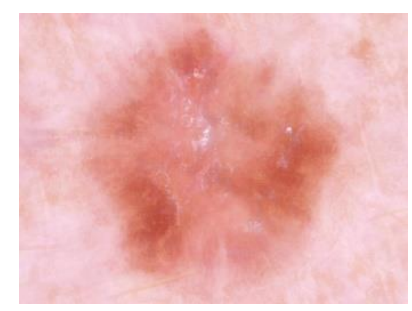

(c)

Figure 1. Selected types of skin cancer [28]: (a) Melanoma, (b) Basal cell carcinoma, (c) Squamous cell carcinoma

\subsection{Data pre-processing}

Data pre-processing is the essential step when dealing with complex skin cancer images and also able to provide the good classification with less testing or training time. Without pre-processing step, there will be the major problem in the feature extraction step as the prominent features unable to produce and consequently also degrades the overall performance. This is because skin cancer images taken from a normal camera may contains the fine hair that may influence the outcome of the study. Therefore, the skin cancer images that contains fine hair removed by using hair removal technique through open-source computer vision library (OpenCV) approach in this paper. Figure 2 illustrates the process of hair removal technique through OpenCV.

Initially, the original skin cancer images in Figure 2(a) that contain fine hair convert to the grayscale Figure 2(b) and then perform the morphological black hat operation. This operation is to enhance the skin cancer image that contain the fine hair which are smaller than the structuring element and darker than their surroundings. Therefore, the fine hair contour will be highlighted Figure 2(c) and the intensity of the fine hair 
contour will be enhanced by using image thresholding method Figure 2(d). The fine hair contour will be removed and inpaint the original image to produce the clean skin cancer images without fine hair Figure 2(e).

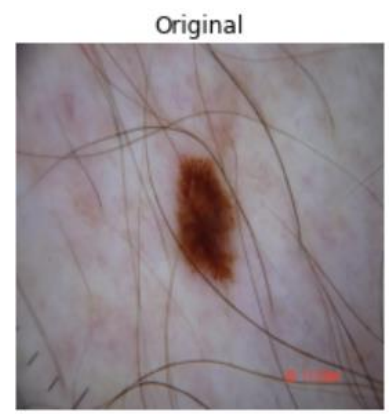

(a)

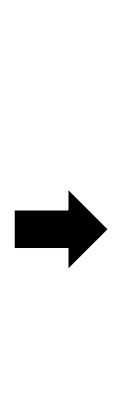

Threshold

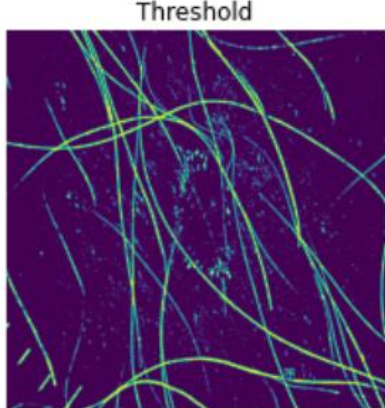

(d)

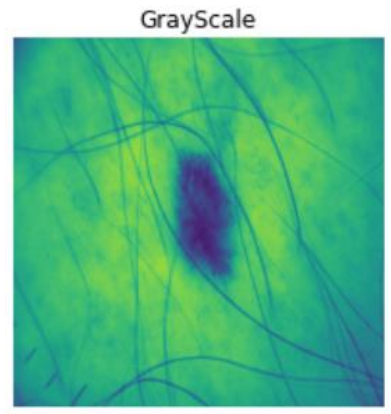

(b)

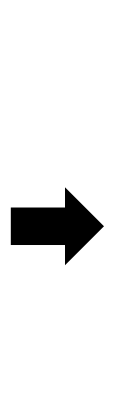

Final_image

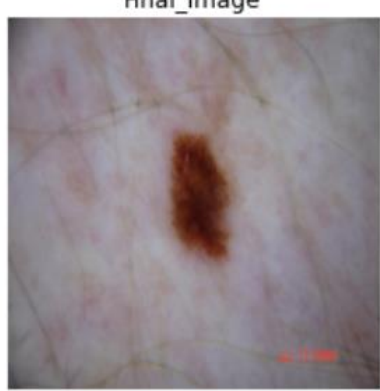

(e)

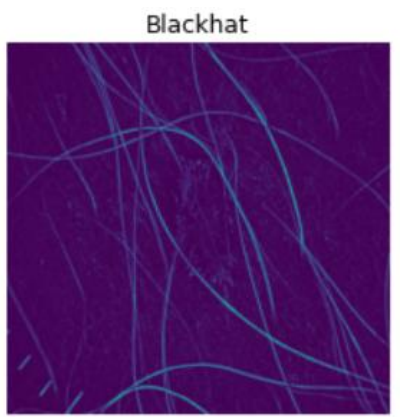

(c)

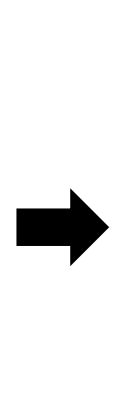

Figure 2. Hair removal process using OpenCV for original basal cell carcinoma image: (a) Original basal cell carcinoma, (b) Grayscale image, (c) After morphological black hat operation,

(d) Thresholded image, (e) Finalized image without fine hair

\subsection{Data augmentation}

There are two purposes of data augmentation technique utilized in this paper such as to increase the dataset and to avoid overfit or underfit issues that might result in poor accuracy. Initially, there were total of 995 original skin cancer images collected through Kaggle which the data collected was limited but more dataset was needed for better performance. There is no specific requirement for deep learning but the training data set must be in high quality in medical imaging [29]. Based on [30], at least 500 to more than 1000 images per class is good enough for classification task in deep neural network [31]. Therefore, the dataset used in this paper have been increased to the total of 4000 images that consist of the pre-processed images without fine-hair and the augmented images which utilized via the intensity adjustment technique from original image to blur, light or dark in order to generate additional data to establish a class-balanced dataset. The number of images before and after augmentation task was given in Table 1.

Table 1. Skin cancer images before and after augmentation

\begin{tabular}{ccc}
\hline Skin cancer & Before augmentation & After augmentation \\
\hline Basal Cell Carcinoma & 376 & 1333 \\
Melanoma & 438 & 1334 \\
Squamous Cell Carcinoma & 181 & 1333 \\
Total & 995 & 4000 \\
\hline
\end{tabular}

After that, the further augmented approaches such as width shift, height shift, zoom, stretch and rotation were selected to avoid overfit or underfit issues help the model to generalize properly. This process able to provide the data set with acceptable size. The detail parameters choice for further data augmentation of skin cancer datasets were summarized in Table 2. 


\subsection{CNN-ARIMA architecture}

In this paper, the performance for the hybrid (the combined architecture) of CNN and ARIMA will be investigated for skin cancer classification. The idea of this hybridization is CNN algorithm behave as feature extractor while ARIMA model acts as classifier by merging into fully connected layer which performs non-linear transformations of the extracted features as the enhancement for classification. As compare to the traditional CNN algorithm, the fully connected layer in this architecture will be modified by inserting ARIMA model in this layer as shown in Figure 3 to output three skin cancer classes as proposed.

Table 2. Data augmentation parameters and values before training

\begin{tabular}{ccc}
\hline Data augmentation parameters & Value of parameter & Action \\
\hline width_shift_range & 0.1 & Randomly shifts the image size in vertical direction by 0.2 \\
height_shift_range & 0.1 & Randomly shifts the image size in horizontal direction by 0.2 \\
zoom_range & 0.2 & Zoom from the center by factor of 0.2 \\
shear_range & 0.1 & Stretch the image by factor of 0.1 \\
rotation_range & 10 & Rotate from - 10 to 10 \\
\hline
\end{tabular}

Before preprocessed and augmented dataset implement in the CNN-ARIMA model, all of these datasets need to resize to the shape of $32 \times 32$ and consequently convert every pixel of images to array which known as feature maps. The input will be the skin cancer image with $2 \mathrm{D}$ array that corresponding to the pixel values. According to Figure 3, the proposed CNN-ARIMA model is the multistage architecture consists of three stages that consists of multiple layers and fully connected layers which are connected after these stages. The details of the proposed CNN-ARIMA model are such as:

- First stage - Third Stage: The input initially fed into first stage of the CNN-ARIMA model. The first and second convolutional layers in this stage contain 64 filters with the kernel size of $5 \times 5$ followed by rectified linear unit (ReLU) as an activation function after each convolutional layer. The output size after pooling layer decreases by half since a stride of 2 have been used with pooling size of $2 \times 2$. The dropout layer with the ratio of 0.5 also added after pooling layer to avoid overfitting. The final dropout layer in this stage has an output of $12 \times 12 \times 64$ which will behave as input in next stage. The same step will be repeated in next two stages except the 32 filters with kernel size of $3 \times 3$ and 16 filters with kernel size of $1 \times 1$ are used in second stage and third stage respectively.

- Fully connected layer: The output from final dropout layer in third stage convert to a single array through flatten layer which also means convert the $3 \mathrm{D}$ array into a $1 \mathrm{D}$ array of size $2 \times 2 \times 16=64$. After that, the ARIMA model obtain in the ARIMA layer via the algorithm;

$$
\operatorname{ARIMA}(3,(p, d, q))
$$

Where $p$ is the auto regressive value, $d$ is the differencing value and $q$ is the moving average value. However, whatever value insert in $p, d$ and $q$, the output parameter still the same which will not affect the performance in this study. In the final last 3 layers, the dense implement according to large 512-unit layers with the dropout ratio of 0.5 followed by the final layer with 3 nodes for classification performance among 3 classes of skin cancer which are basal cell carcinoma, melanoma and squamous cell carcinoma through a softmax activation function.

In addition, there are also the parameters compute after every layer in model except input layer, dropout layer and flatten layer due to there is no backpropagation learning involve in these layers. The number of parameters for convolutional layer, Param $_{c o n v}$ and fully connected layer, Param fully are computed by the formula;

$$
\begin{aligned}
& \operatorname{Param}_{\text {conv }}=((m \times n \times f)+1) \times k \\
& \text { Param }_{f u l l y}=(l+1) \times c
\end{aligned}
$$

Where $m$ is the shape of width of the filter, $n$ is the shape of height of the filter, $f$ is the number of filters in the previous layer, $k$ is the number of filters in current layer, $l$ is the previous layer neurons and $c$ is the current layer neurons. The total parameter computed for the proposed CNN-ARIMA model as shown in Table 3 is 136422 which also based on the sum of every single parameter from each layer. 
Table 3. Summary of proposed CNN-ARIMA architecture

\begin{tabular}{lcll}
\hline \multicolumn{1}{c}{ Layer (type) } & Output shape & Output size & Parameters \\
\hline input_1 (Input) & $(32,32,1)$ & 1024 & 0 \\
& First Stage & & \\
conv2d_1 (Conv2D) & $(28,28,64)$ & 50176 & 1664 \\
conv2d_2 (Conv2D) & $(24,24,64)$ & 36864 & 102464 \\
max_pooling2d_1 (MaxPooling2D) & $(12,12,64)$ & 9216 & 0 \\
dropout_1 (Dropout) & $(12,12,64)$ & 9216 & 0 \\
& Second Stage & & \\
conv2d_3 (Conv2D) & $(10,10,32)$ & 3200 & 18464 \\
conv2d_4 (Conv2D) & $(8,8,32)$ & 2048 & 9248 \\
max_pooling2d_2 (MaxPooling2D) & $(4,4,32)$ & 512 & 0 \\
dropout_2 (Dropout) & $(4,4,32)$ & 512 & 0 \\
& Third Stage & & \\
conv2d_5 (Conv2D) & $(4,4,16)$ & 256 & 528 \\
conv2d_6 (Conv2D) & $(4,4,16)$ & 256 & 272 \\
max_pooling2d_3 (MaxPooling2D) & $(2,2,16)$ & 64 & 0 \\
dropout_3 (Dropout) & $(2,2,16)$ & 64 & 0 \\
& & \\
flatten_1 (Flatten) & Fully Connected Layer & & \\
arima_1 (ARIMA) & $(64,1)$ & 64 & 0 \\
dense_1 (Dense) & $(3,1)$ & 3 & 195 \\
dropout_4 (Dropout) & $(512,1)$ & 512 & 2048 \\
dense_2 (Dense) & $(512,1)$ & 512 & 0 \\
output_1 (Output) & $(3,1)$ & 3 & 1539 \\
Total Trainable Parameters & $(3,1)$ & 3 & 0 \\
\hline & & & 136422 \\
\hline
\end{tabular}

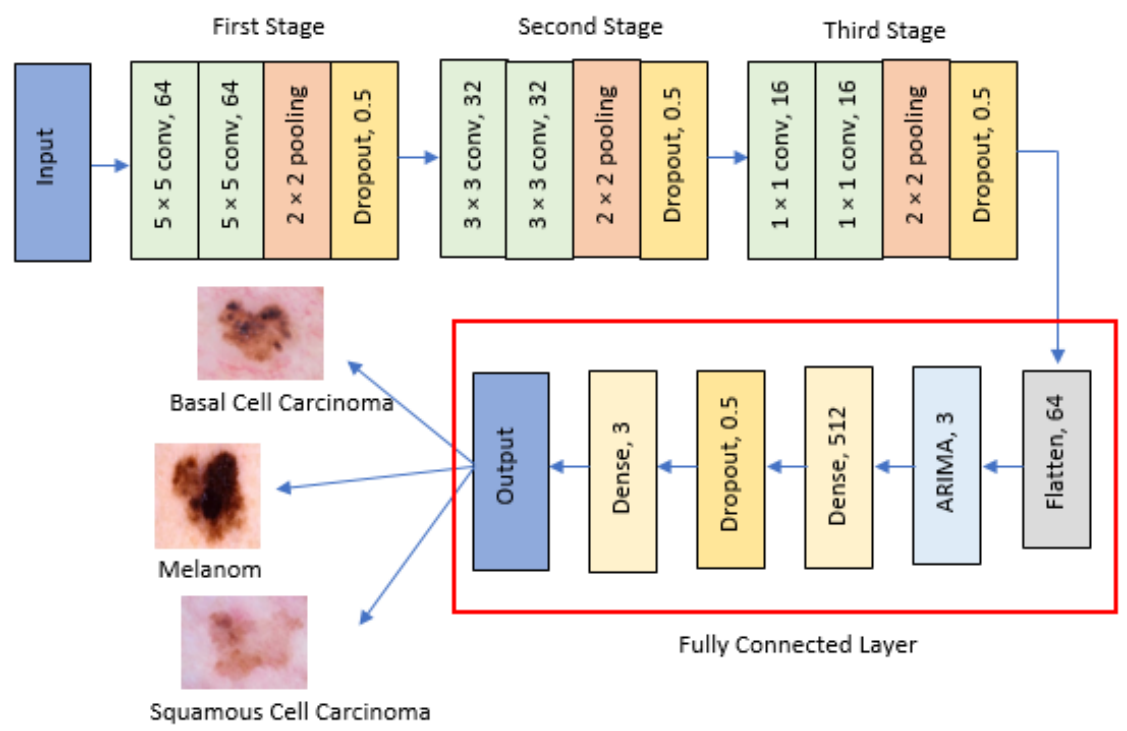

Figure 3. CNN-ARIMA architecture

\subsection{Training the model}

The image data split into the ratio of 70:30 for training and testing dataset respectively. Meanwhile, $30 \%$ of training data will be further utilized for validation purpose. The testing dataset will also balance distributed randomly to avoid overfitting. The important parameters such as batch size, epoch values and learning rate needed to be concerned in the training of the proposed CNN-ARIMA model. These values were 64 for batch size, 30 epoch values with 2000 steps per epoch and learning rate of 0.0001 . Furthermore, Adam was utilized for neural work optimization and categorical cross entropy [32] was behave as loss function in this training of proposed CNN-ARIMA model. For the model implementation, this proposed model was run through tensorflow (2.2.0) and Keras (2.3.1).

\subsection{Performance measure}

The classification result for the proposed model has been evaluated in four quantitative measures such as test accuracy (ACC), sensitivity (TPR), specificity (TNR) and precision (PPV) via confusion matrix [33]. These measures are determined by the formula [34]; 


$$
\begin{aligned}
& A C C=\frac{T P+T N}{T P+T N+F P+F N} \times 100 \% \\
& T P R=\frac{T P}{T P+F N} \times 100 \% \\
& T N R=\frac{T N}{T N+F P} \times 100 \% \\
& P P V=\frac{T P}{T P+F P} \times 100 \%
\end{aligned}
$$

Where $T P$ is true positive value, $T N$ is true negative value, $F P$ is false positive value and $F N$ is false negative value while $T P R, T N R$ and $P P V$ refers to true positive rate, true negative rate and positive prediction value respectively. Therefore, the classification result performance in this paper consists of overall test accuracy with the average sensitivity, average specificity and average precision according to basal cell carcinoma, melanoma and squamous cell carcinoma.

\section{RESULTS AND DISCUSSION}

In this experiment, the test accuracy achieved for the proposed CNN-ARIMA model is $92.25 \%$. From the loss and accuracy performance for training and validation respectively as shown in Figure 4, the flow of training was decrease exponentially for loss performance while increase exponentially for accuracy performance which able to produce high accuracy. After $14^{\text {th }}$ epoch during training and validating in loss performance, the validation loss is higher than training loss as shown in Figure 4(a). This show that the model was slightly overfit after $14^{\text {th }}$ epoch due to the model only able to memorize the limited amount of training data even the data augmentation technique has been implemented and testing dataset have been balance distributed randomly in this work. Meanwhile, the validation accuracy is lower than training accuracy after $15^{\text {th }}$ epoch in accuracy performance as shown in Figure 4(b) due to the slightly underfit issue. This shows that the validation data may perform well under circumstances which the validation data fits better to the model than training data even though the data is in underfitting situation.

Based on the confusion matrix performance as shown in Figure 5, majority of skin cancer types were classified accurately since the test accuracy shows the promising performance. The 1200 testing images (30\% of overall dataset) has been balance distributed randomly in three types of skin cancer which 415 images distributed in basal cell carcinoma, 402 images distributed in melanoma and 383 images distribute in squamous cell carcinoma. From the balance distributed testing dataset in each skin cancer, 362 images of basal cell carcinoma, 371 images of melanoma and 374 images of squamous cell carcinoma were categorized accurately in confusion matrix performance as shown in Figure 5.

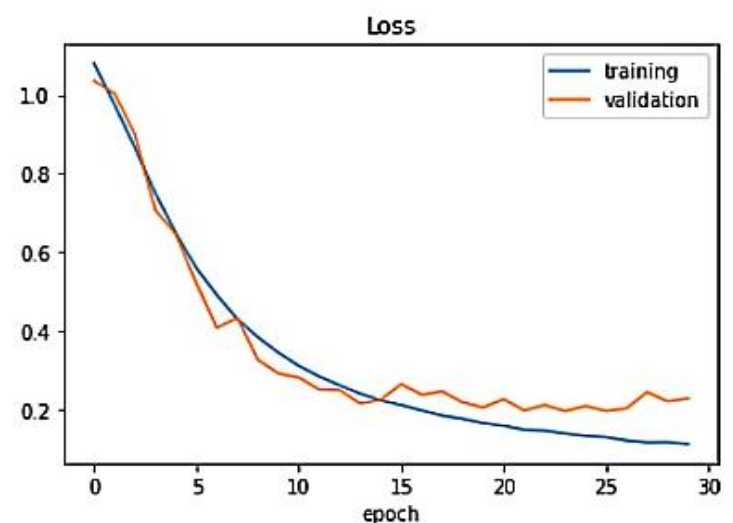

(a)

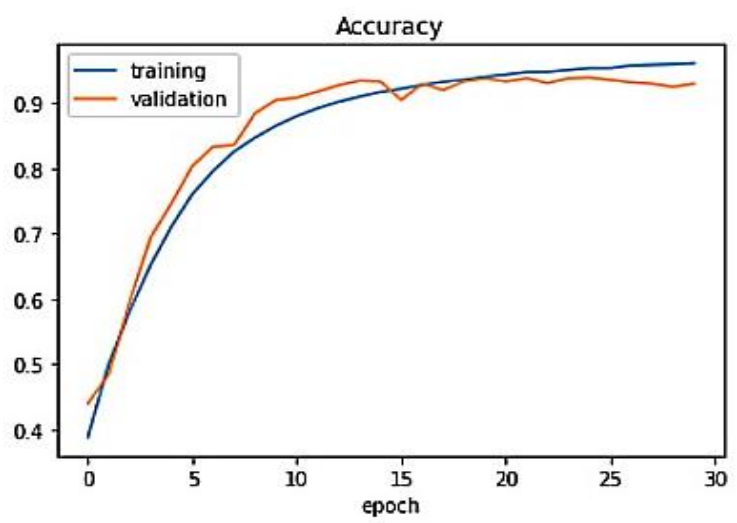

(b)

Figure 4. Training and validation performance: (a) Loss, (b) Accuracy 


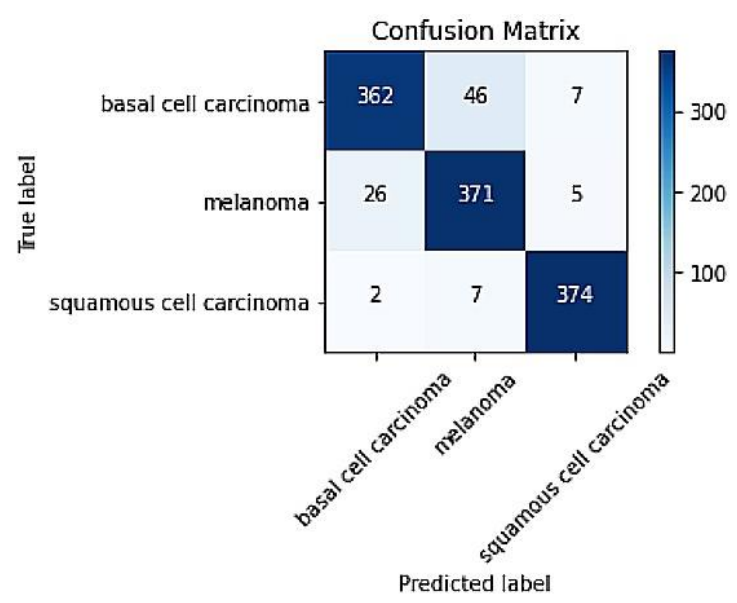

Figure 5. The confusion matrix of the classification accuracy for the proposed CNN-ARIMA model

According to Table 4, the performance measure of three skin cancer types via confusion matrix as shown in Figure 5 achieve the average sensitivity of $92.39 \%$, average specificity of $96.11 \%$ and average precision of $92.40 \%$. In addition, the performance of the proposed CNN-ARIMA model also compared with the existing techniques using the different amount of skin cancer dataset and different classes of skin cancer to classify. Results of comparative study as presented in Table 5 show that the proposed CNN-ARIMA model achieve better performance than the existing tehniques in the term of sensitivity and precision, as it has been able to accurately distinguish skin cancer in various classes of skin cancer. However, the classification accuracy and specifity for the proposed model of CNN-ARIMA is still marginally lower than the previous work proposed by Kassem et al. [22].

Table 4. Performance measure for every type of skin cancer

\begin{tabular}{cccc}
\hline Type of skin cancer & Sensitivity & Specificity & Precision \\
\hline Basal Cell Carcinoma & $87.23 \%$ & $96.43 \%$ & $92.82 \%$ \\
Melanoma & $92.29 \%$ & $93.36 \%$ & $87.50 \%$ \\
Squamous Cell Carcinoma & $97.65 \%$ & $98.53 \%$ & $96.89 \%$ \\
Average & $92.39 \%$ & $96.11 \%$ & $92.40 \%$ \\
\hline
\end{tabular}

Table 5. Comparative study

\begin{tabular}{cccccc}
\hline Methods & Classification technique & TPR & TNR & PPV & ACC \\
\hline Ozkan and Koklu [17] & ANN & $90.86 \%$ & $96.11 \%$ & $92.38 \%$ & $92.50 \%$ \\
Oliveira et al. [18] & SVM & - & - & $78.89 \%$ & $79.01 \%$ \\
T. Emara [19] & Modified Inception-v4 & $71.70 \%$ & $95.80 \%$ & - & $94.70 \%$ \\
Mukherjee et al. [21] & CMLD & - & - & - & $90.58 \%$ \\
Kassem et al. [22] & GoogleNet-SVM & $79.80 \%$ & $97.00 \%$ & $80.36 \%$ & $94.92 \%$ \\
Proposed Method & CNN-ARIMA & $92.39 \%$ & $96.11 \%$ & $92.40 \%$ & $92.25 \%$ \\
\hline
\end{tabular}

\section{CONCLUSION}

This research introduced the hybrid algorithm of deep neural network and machine learning approach for skin cancer classification. The hybrid approach was relied on the combination of CNN and ARIMA. The proposed hybrid performance shown significantly higher results in terms of sensitivity, specificity and precision as compared to the state-of-art skin cancer classification approaches that used single machine learning and single deep learning methods. In addition, this work also did not require any prior segmentation approach.

\section{ACKNOWLEDGEMENTS}

It is hereby declared that, this paper has not been submitted anywhere for publication and there is no conflict of interest for publishing this paper. Authors would like to thank University Malaysia Sarawak 
(UNIMAS) and Fusionex International for supporting this research through UNIMAS-Fusionex Research Grant (RG/F02/FUSX/01/2019).

\section{REFERENCES}

[1] Beacon Hospital, "Skin Cancer," 2016. [Online] Available: http://www.beaconhospital.com.my/skin-cancer/ [Accessed Dec. 10. 2018]., p. 2018, 2018.

[2] A. M. Affandi, "Is skin cancer common in Malaysia?" The Star Online, Feb. 2015. [Online], Available https://www.thestar.com.my. [Accessed Dec. 10, 2018]., p. 2018, 2018.

[3] Z. Khazaei, M. Sohrabivafa, K. Mansori, H. Naemi, and E. Goodarzi, "Incidence and mortality of cervix cancer and their relationship with the human development index in 185 countries in the world: An ecology study in 2018," Advances in Human Biology, vol. 9, no. 3, p. 222, 2019, doi: 10.4103/aihb.aihb_15_19.

[4] M. Kunz and W. Stolz, "ABCD rule," Dermoscopedia Organization, 17 Jan. 2018. Available: https://dermoscopedia.org/ABCD_rule. [Accessed 11 Nov. 2020].,"p. 2020, 2020.

[5] B. Tas, "Faster Evaluation of ABCD Rule and Total Dermoscopic Score for Nevomelanocytic Lesions: Dermoscopic Score Scale," JOURNAL OF SKIN AND STEM CELL, vol. 6, no. 2, 2020, doi: 10.5812/jssc.102138.

[6] P. Carli et al., "Education Pattern analysis, not simplified algorithms, is the most reliable method for teaching dermoscopy for melanoma diagnosis to residents in dermatology," British Journal of Dermatology, vol. 148, no. 5, pp. 981-984, 2003, doi: 10.1046/j.1365-2133.2003.05023.x.

[7] J. Kawahara, S. Daneshvar, G. Argenziano, and G. Hamarneh, "Seven-Point Checklist and Skin Lesion Classification Using Multitask Multimodal Neural Nets," in IEEE Journal of Biomedical and Health Informatics, vol. 23, no. 2, pp. 538-546, March 2019, doi: 10.1109/JBHI.2018.2824327.

[8] R. Garnavi, M. Aldeen, and J. Bailey, "Computer-Aided Diagnosis of Melanoma Using Border- and Wavelet-Based Texture Analysis," in IEEE Transactions on Information Technology in Biomedicine, vol. 16, no. 6, pp. 1239-1252, Nov. 2012, doi: 10.1109/TITB.2012.2212282.

[9] F. Kaliyadan, "The scope of the dermoscope," Indian Dermatol. Online J., vol. 7, no. 5, p. 359, 2016, doi: 10.4103/2229-5178.190496.

[10] T. Tiwari, T. Tiwari, and S. Tiwari, "How Artificial Intelligence, Machine Learning and Deep Learning are Radically Different?," International Journal of Advanced Research in Computer Science and Software Engineering, vol. 8, no. 2, p. 1, 2018, doi: 10.23956/ijarcsse.v8i2.569.

[11] J. Vincent, "Artificial intelligence can spot skin cancer as well as a trained doctor," The Verge, p. 14396500, 2019.

[12] S. Loussaief and A. Abdelkrim, "Convolutional Neural Network hyper-parameters optimization based on Genetic Algorithms," International Journal of Advanced Computer Science and Applications, vol. 9, no. 10, pp. 252-266, 2018, doi: 10.14569/IJACSA.2018.091031.

[13] G. E. P. Box, G. M. Jenkins, G. C. Reinsel, and G. M. Ljung, "Time Series Analysis: Forecasting and Control," Journal of Time Series Analysis, vol. 37, no. 5, pp. 712, March 2016, doi: 10.1111/jtsa.12194.

[14] M. A. F. Quioc, S. C. Ambat, and A. C. Lagman, "Medical Cases Forecasting for the Development of Resource Allocation Recommender System," 2019 IEEE 4th International Conference on Computer and Communication Systems (ICCCS), 2019, pp. 414-418, doi: 10.1109/CCOMS.2019.8821673.

[15] N. C. B, J. Cai, M. Abedini, and R. Garnavi, "Deep Learning, Sparse Coding, and SVM for Melanoma Recognition in Dermoscopy Images," International Workshop on Machine Learning in Medical Imaging, vol. 1, pp. 118-126, 2015.

[16] N. C. F. Codella et al., "Deep learning ensembles for melanoma recognition in dermoscopy images 1 Dataset," vol. 61, no. 4, pp. 1-28, 2017.

[17] M. Koklu and I. A. Ozkan, "Skin Lesion Classification using Machine Learning Algorithms," International Journal of Intelligent Systems and Applications in Engineering, vol. 4, no. 5, pp. 285-289, 2017, doi: 10.18201/ijisae.2017534420.

[18] R. B. Oliveira, N. Marranghello, A. S. Pereira, and J. M. R. S. Tavares, "A computational approach for detecting pigmented skin lesions in macroscopic images," Expert Systems with Applications, vol. 61, pp. 53-63, 2016, doi: 10.1016/j.eswa.2016.05.017.

[19] T. Emara, H. M. Afify, F. H. Ismail and A. E. Hassanien, "A Modified Inception-v4 for Imbalanced Skin Cancer Classification Dataset," 2019 14th International Conference on Computer Engineering and Systems (ICCES), 2019, pp. 28-33, doi: 10.1109/ICCES48960.2019.9068110.

[20] P. Tschandl, C. Rosendahl, and H. Kittler, "The HAM10000 dataset, a large collection of multi-source dermatoscopic images of common pigmented skin lesions," Scientific Data, vol. 5, no. 1, pp. 1-9, 2018, doi: 10.1038/sdata.2018.161.

[21] S. Mukherjee, A. Adhikari, and M. Roy, "Malignant Melanoma Classification Using Cross-Platform Dataset with Deep Learning CNN Architecture," Recent Trends in Signal and Image Processing, pp. 31-41, 2019, doi: 10.1007/978-981-13-6783-0.

[22] M. A. Kassem, K. M. Hosny, and M. M. Fouad, "Skin Lesions Classification into Eight Classes for ISIC 2019 Using Deep Convolutional Neural Network and Transfer Learning," IEEE Access, vol. 8, pp. 114822-114832, 2020, doi: 10.1109/ACCESS.2020.3003890.

[23] B. Wang et al., "Research on hybrid model of garlic short-term price forecasting based on big data," Computers, Materials and Continua, vol. 57, no. 2, pp. 283-296, 2018, doi: 10.32604/cmc.2018.03791.

[24] T. Ma, C. Antoniou, and T. Toledo, "Hybrid machine learning algorithm and statistical time series model for network-wide traffic forecast," Transportation Research Part C: Emerging Technologies, vol. 111, pp. 352-372,

Hybrid of convolutional neural network algorithm and autoregressive integrated... (Chee Ka Chin) 
2020, doi: $10.1016 /$ j.trc.2019.12.022.

[25] N. Suhermi, D. D. Prastyo, and B. Ali, "Roll motion prediction using a hybrid deep learning and ARIMA model," Procedia Computer Science, vol. 144, pp. 251-258, 2018, doi: 10.1016/j.procs.2018.10.526.

[26] L. Ji, Y. Zou, K. He, and B. Zhu, "Carbon futures price forecasting based with ARIMA-CNN-LSTM model," vol. 162, pp. 33-38, 2019, doi: 10.1016/j.procs.2019.11.254.

[27] Z. Apalla, A. Lallas, E. Sotiriou, E. Lazaridou, and D. Ioannides, "Epidemiological trends in skin cancer: What Does the Future Hold," Dermatology practical \& conceptual, vol. 7, no. 2, pp. 1-6, 2017, doi: $10.5826 /$ dpc.0702a01.

[28] https://www.kaggle.com/nodoubttome/skin-cancer9-classesisic.

[29] J. Cho, K. Lee, E. Shin, G. Choy, S. Do, and M. G. Hospital, "How Much Data Is Needed To Train A Medical Image Deep Learning System To Achieve Necessary High Accuracy?,” 2016.

[30] A. Sarkis, "How to create AI ready data for object detection," [online] Available, "https://medium.com\%2F@medium.com/diffgram/how-to-create-ai-ready-data-for-object-detectione395f9dc6cb9," p. 395.

[31] R. N. D'souza, P. Y. Huang, and F. C. Yeh, "Structural Analysis and Optimization of Convolutional Neural Networks with a Small Sample Size," Scientific Reports, vol. 10, no. 1, pp. 1-13, 2020, doi: 10.1038/s41598-02057866-2.

[32] Y. Ho and S. Wookey, "The Real-World-Weight Cross-Entropy Loss Function: Modeling the Costs of Mislabeling," in IEEE Access, vol. 8, pp. 4806-4813, 2020, doi: 10.1109/ACCESS.2019.2962617.

[33] M. Ali, D. H. Son, S. H. Kang, and S. R. Nam, "An accurate CT saturation classification using a deep learning approach based on unsupervised feature extraction and supervised fine-tuning strategy," Energies, vol. 10, no. 11, 2017, doi: 10.3390/en10111830.

[34] M. Stojanovi et al., "Understanding sensitivity, specificity, and predictive values," Vojnosanitetski Pregled, vol. 71, no11, pp. 1062-1065, 2014, doi: 10.2298/VSP130603026G.

\section{BIOGRAPHIES OF AUTHORS}
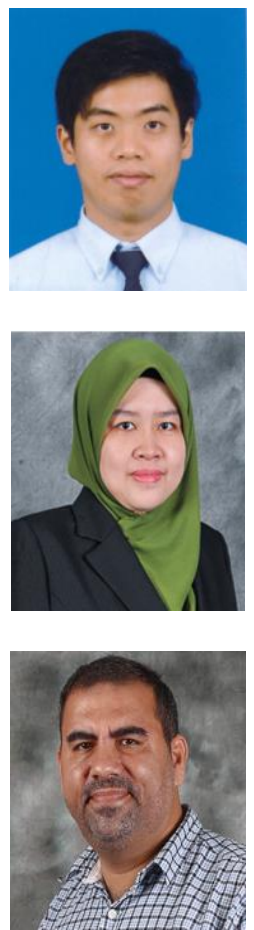

Mr. Chee Ka Chin is currently pursuing the master's degree of engineering in Faculty of Engineering, University Malaysia Sarawak (UNIMAS). His research interests include artificial intelligence and deep learning application.

Dr. Dayang Azra binti Awang Mat is a Senior Lecturer in Faculty of Engineering, University Malaysia Sarawak (UNIMAS) from 2006 until now. She graduated from Kyushu University Japan, in 2014 with Doctor of Engineering (in RF and millimeter-wave design). She received her MEng. (Computer and Communication) in 2006 at Universiti Kebangsaan Malaysia and degree in BEng. (Hons) Electronic and Telecommunication at Universiti Malaysia Sarawak. She is expert in mobile and wireless communication, antenna and filter design, RF both in microwave and millimeter-wave.

Dr. Abdulrazak Yahya Saleh is a Senior Lecturer in Faculty of Cognitive Sciences and Human Development, Universiti Malaysia Sarawak (UNIMAS). He received his PhD from Universiti Teknologi Malaysia, MSc and BSc from University of Science and Technology, Yemen. His research is mainly on the cognitive science, artificial intelligence, neural networks, spiking neural networks, brain modeling, optimization methods, multi-objective optimization algorithms and machine learning techniques and their applications. The current focus of his research is on deep learning applications and challenges in big data analytics. 